\title{
The Epidemiology and Pathogenesis and Treatment of Pseudomonas aeruginosa Infections: An Update
}

\author{
Dan Reynolds ${ }^{1} \cdot$ Marin Kollef $^{1}$ (D) \\ Accepted: 17 October 2021 / Published online: 7 November 2021 \\ (c) The Author(s), under exclusive licence to Springer Nature Switzerland AG 2021
}

\begin{abstract}
Pseudomonas aeruginosa is a Gram-negative bacterial pathogen that is a common cause of nosocomial infections, particularly pneumonia, infection in immunocompromised hosts, and in those with structural lung disease such as cystic fibrosis. Epidemiological studies have identified increasing trends of antimicrobial resistance, including multi-drug resistant (MDR) isolates in recent years. P. aeruginosa has several virulence mechanisms that increase its ability to cause severe infections, such as secreted toxins, quorum sensing and biofilm formation. Management of $P$. aeruginosa infections focuses on prevention when possible, obtaining cultures, and prompt initiation of antimicrobial therapy, occasionally with combination therapy depending on the clinical scenario to ensure activity against $P$. aeruginosa. Newer anti-pseudomonal antibiotics are available and are increasingly being used in the management of MDR P. aeruginosa.
\end{abstract}

\section{Key Points}

Pseudomonas aeruginosa is an important Gram-negative organism that is implicated in nosocomial infections, and in patients with structural lung disease, such as in cystic fibrosis.

$P$. aeruginosa attacks respiratory epithelial cells via its pili and flagellum, and utilizes biofilm formation, quorum sensing, and a type III secretion system to increase its virulence.

Newer antimicrobials that have activity against MDR $P$. aeruginosa have been shown to be efficacious in the treatment of MDR Pseudomonas.
Marin Kollef

mkollef@im.wustl.edu

1 Division of Pulmonary and Critical Care Medicine, Washington University School of Medicine, St. Louis, USA

\section{Introduction}

Pseudomonas aeruginosa is a Gram-negative pathogen that is commonly associated with hospital-acquired infections, infections in immunocompromised hosts, and chronic infections in patients with structural lung disease such as cystic fibrosis (CF). For this review, we sought to update the readers with regard to the epidemiology of $P$. aeruginosa infections, mechanisms of virulence of $P$. aeruginosa, which aid in its ability to cause severe infection, and advancements in the diagnosis and management of $P$. aeruginosa. The authors conducted an extensive literature review utilizing the MEDLINE/Pubmed and Cochrane library databases, aiming to find relevant articles on $P$. aeruginosa epidemiology, pathogenesis, treatment, nosocomial infections, hospital-acquired and ventilator associated pneumonias, as well as CF.

\section{Epidemiology}

\subsection{Pseudomonas aeruginosa and Nosocomial Infections}

$P$. aeruginosa is a common cause of nosocomial infections, manifesting as pneumonia, surgical site infections, urinary tract infections and bacteremia. It is estimated that $P$. aeruginosa has a prevalence of $7.1 \%-7.3 \%$ amongst all healthcareassociated infections $[1,2]$. The most common site of $P$. 
aeruginosa infection is pneumonia, and it is the most common Gram-negative organism identified in nosocomial pneumonia. Prevalence has been increasing over the past decade $[3,4]$. In intensive care unit (ICU) patients, $P$. aeruginosa is responsible for an even higher percentage of healthcareassociated infections. A large international observational point-prevalence study of infections in ICU patients found that $P$. aeruginosa represented $16.2 \%$ of patient infections and was the cause of $23 \%$ of all ICU-acquired infections, with a respiratory source being the most common site of $P$. aeruginosa infection [5].

Healthcare-associated pneumonia (HAP) and ventilatorassociated pneumonia (VAP) are a significant source of stress on the healthcare system, and they account for up to $22 \%$ of all healthcare-acquired infections [1, 6]. P. aeruginosa accounts for $10 \%-20 \%$ of isolates in cases of VAP, second only to Staphylococcus aureus [2]. Nosocomial pneumonia secondary to $P$. aeruginosa is associated with worse outcomes than other typical organisms [7-9]. Mortality from VAP secondary to $P$. aeruginosa is estimated to be as high as $32 \%-42.8 \%[7,10,11]$. One multicenter observational study estimated the global prevalence of VAP due to $P$. aeruginosa as $4.1 \%$, and $P$. aeruginosa was the most common cause of VAP globally, accounting for $26 \%$ of cases, with the most common risk factors for $P$. aeruginosa VAP being prior colonization with $P$. aeruginosa and prolonged hospitalization [12]. A prospective observational study of 28 ICUs in the USA estimated that $P$. aeruginosa was the cause of $11 \%$ of all HAP/VAP in ICU patients deemed to be at risk for developing nosocomial pneumonia, second only to $S$. aureus [13].

P. aeruginosa is a common cause of nosocomial urinary tract infections (UTI), particularly catheter-associated UTI (CAUTI). P. aeruginosa accounts for approximately $10 \%$ of all CAUTIs, and up to $16 \%$ of UTIs in ICU patients [14, 15]. Nosocomial UTI secondary to P. aeruginosa is associated with high morbidity and mortality and bacteremia is a potential complication [16-18]. Additionally, $P$. aeruginosa CAUTI is associated with high rates of antimicrobial resistance, depending on local antimicrobial resistance patterns. Data from the International Nosocomial Infection Control Consortium report found resistance rates of $>40 \%$ for antibiotics such as fluoroquinolones, piperacillin-tazobactam, and meropenem in ICU patients, although the report notes these resistance rates are higher than are reported elsewhere [14].

Amongst healthcare-associated infections reported to the National Healthcare Safety Network at the Centers for Disease Control and Prevention (CDC) from 2011-2014, 5.7\% of surgical site infections were found to be secondary to $P$. aeruginosa, with breast and cardiac surgeries being the type of surgery most associated with P. aeruginosa [2]. Data from England from 2000-2013 showed that Pseudomonas species accounted for $4.3 \%-6.5 \%$ of all surgical site infection (SSI) annually [19]. Additionally, $P$. aeruginosa infection after surgery is associated with worse outcomes, with a retrospective study of cardiac surgeries performed at a university hospital over a 7 -year period showing that $P$. aeruginosa infection was associated with increased mortality [20].

P. aeruginosa is a known complication of and important pathogen in burn patients, with the moist environment of burn-wound patients believed to contribute to its predilection for burn patients [21]. P. aeruginosa is the most common Gram-negative organism leading to infection in burnwound patients, and it is associated with sepsis and death [21-24]. Multi-drug resistant (MDR) P. aeruginosa is an increasingly common cause of death in burn patients, with $86 \%$ of sepsis deaths in a pediatric burn ICUs due to MDR organisms, with $P$. aeruginosa as the responsible organism $64 \%$ of the time from 1999-2009 [22].

Bloodstream infections (BSIs) due to P. aeruginosa are associated with high rates of morbidity and mortality, with estimated mortality rates of $43.2 \%-58.8 \%$ [25-27]. Additionally, MDR BSIs of P. aeruginosa are estimated to complicate $16.7 \%-28 \%$ of $P$. aeruginosa BSIs, with increased mortality associated with the presence of MDR P. aeruginosa [26, 27]. Furthermore, P. aeruginosa BSI was more likely to be a hospital-acquired bacteremia compared to all other Gram-negative BSIs (51\% vs $27 \%$ ) in a 13 -year study of all BSIs in a large university medical center [27]. P. aeruginosa is estimated to represent $4.0 \%$ of all central lineassociated BSIs [2].

\subsection{Cystic Fibrosis and P. aeruginosa}

In patients with cystic fibrosis $(\mathrm{CF}), P$. aeruginosa is a critically important pathogen, and is a predominant cause of morbidity and mortality [28]. Complications of CF include chronic respiratory infections, structural lung disease and bronchiectasis, airflow obstruction, and death. Chronic infection with $P$. aeruginosa is associated with worsening lung function and guidelines recommend aggressive early therapy to treat $P$. aeruginosa in CF patients in order to preserve lung function [29-35]. P. aeruginosa may thrive in the CF lung due to its ability to undergo mutations and genetic changes that allow it to survive in the typically anaerobic environment of the CF lung [36]. Chronic infection with $P$. aeruginosa typically leads to production of a biofilm, and this conversion to a mucoid phenotype is associated with the production of the polysaccharide alginate [3, 36, 37]. The development of a mucoid phenotype of $P$. aeruginosa is associated with a decline in lung function and is associated with increased mortality, which has led to early treatment of $P$. aeruginosa in CF patients becoming the standard of care [34-36, 38]. 
Aggressive treatment regimens for $P$. aeruginosa has been associated with a decrease in the prevalence of $P$. aeruginosa in CF patients over recent years; however, data from the Cystic Fibrosis Foundation Patient Registry from 2006 to 2012 showed that $P$. aeruginosa still had a prevalence of $49.6 \%$, with increasing prevalence in older patients, as $74.1 \%$ of cultures were positive in patients aged $>26$ years [39]. Data from the European Cystic Fibrosis Society registry showed $P$. aeruginosa had a prevalence of $29.8 \%$ with over half of patients aged $>40$ years having a positive culture for $P$. aeruginosa [40].

\subsection{Non-cystic Fibrosis Bronchiectasis and $P$. aeruginosa}

Bronchiectasis is characterized by the dilation and thickening of airways, which leads to chronic infection and airway inflammation. Non-CF bronchiectasis is more common than $\mathrm{CF}$ and has a variety of causes such as post-infectious, primary ciliary dyskinesia, immunodeficiency, and idiopathic bronchiectasis [41]. P. aeruginosa is one of the most frequently isolated organisms in patients with non-CF bronchiectasis and is associated with worsening lung function and increased mortality [42-44]. Management of $P$. aeruginosa in non-CF bronchiectasis typically consists of treating exacerbations to prevent decline in lung function [44].

\subsection{Immunocompromised Hosts}

$P$. aeruginosa is an especially important pathogen in immunocompromised patients, particularly patients with neutropenia. It is a critically important pathogen in patients with hematological malignancies $[45,46]$. In one multicenter study, $P$. aeruginosa was found to cause $17 \%$ of all Gramnegative BSI in patients with hematological malignancies, with risk factors for $P$. aeruginosa being prior surgery, neutropenia, use of steroids, and severity of underlying disease [45]. Another study found that of all BSIs in patients with hematological malignancies, $P$. aeruginosa was responsible for $12.1 \%$ of all Gram-negative BSI, while also showing an increasing trend of MDR isolates in patients with hematological malignancies [47].

Patients who have undergone transplantation are also at high risk of $P$. aeruginosa infection and are at increased risk of adverse outcomes. Previous mortality estimates of BSI with $P$. aeruginosa in patients with a history of stem cell, liver, or lung transplant have been as high as 50\% [48]. In a large study of over 500 patients with $P$. aeruginosa BSI at a single center, mortality was found to be $42 \%$, with resistance to all antibiotic classes being higher in the transplant patients compared to nontransplant patients. The risk for $P$. aeruginosa is highest immediately after transplantation, with over half (52\%) of $P$. aeruginosa occurring within 3 months of transplant [48].

\subsection{Emerging Resistance Profiles}

Antimicrobial resistance to $P$. aeruginosa remains a serious health threat and is a major source of morbidity and mortality, especially in ICUs and long-term care hospitals (LTACHs). MDR $P$. aeruginosa is found in isolates from CAUTIs, BSI, and VAPs. Data from the CDC show that $9 \%$ of $P$. aeruginosa isolates were MDR in 2018, down from 15.7\% in 2011 [49]. Data from the National Healthcare Safety Network from 2015 to 2017 show that antimicrobial resistance to $P$. aeruginosa remains a serious concern. Amongst $P$. aeruginosa isolates in ICU patients, $26.3 \%$ of isolates were resistant to carbapenems, $26.5 \%$ were resistant to extended-spectrum cephalosporins, and $27.1 \%$ were resistant to fluoroquinolones. Additionally, $18.6 \%$ of isolates from ICU patients were classified as MDR, resistant to 3 or more antimicrobials [50]. LTACHs reported $29.9 \%$ of $P$. aeruginosa isolates were MDR, while $11.6 \%$ of isolates from hospital oncology units were MDR [50].

Carbapenem resistance in $P$. aeruginosa can complicate treatment regimens, given how often $P$. aeruginosa is resistant to other antimicrobials. In a study that investigated the prevalence of carbapenem-resistant $P$. aeruginosa isolates over a 4-month period at multiple centers in the USA, $9 \%$ of $P$. aeruginosa isolates were found to be carbapenem resistant. Over $90 \%$ of the patients who had carbapenem-resistant isolates had healthcare exposures prior to their positive culture, emphasizing the relevance of nosocomial infections in $P$. aeruginosa [51].

Antimicrobial resistance in $P$. aeruginosa BSI is especially relevant, given the increased mortality associated with $P$. aeruginosa BSI [26, 27]. One study found that resistance to one antimicrobial for a $P$. aeruginosa BSI was highly correlated with other antimicrobial resistance. For example, $83 \%$ of $P$. aeruginosa BSI isolates that were resistant to piperacillintazobactam were also resistant to ceftazidime, and $67 \%$ were resistant to ciprofloxacin [52]. Another study that investigated all $P$. aeruginosa BSI at a single center over a 13-year period found that $28 \%$ of isolates were MDR, and $15 \%$ were extensively drug resistant [27]. Nosocomial pneumonia due to $P$. aeruginosa has a high incidence of MDR strains, with an international multicenter retrospective study showing that $30.5 \%$ of nosocomial pneumonia secondary to $P$. aeruginosa were MDR-strains, and this was associated with increased inhospital mortality [53]. 
(A) Pseudomonas aeruginosa attacking respiratory epthelial cells
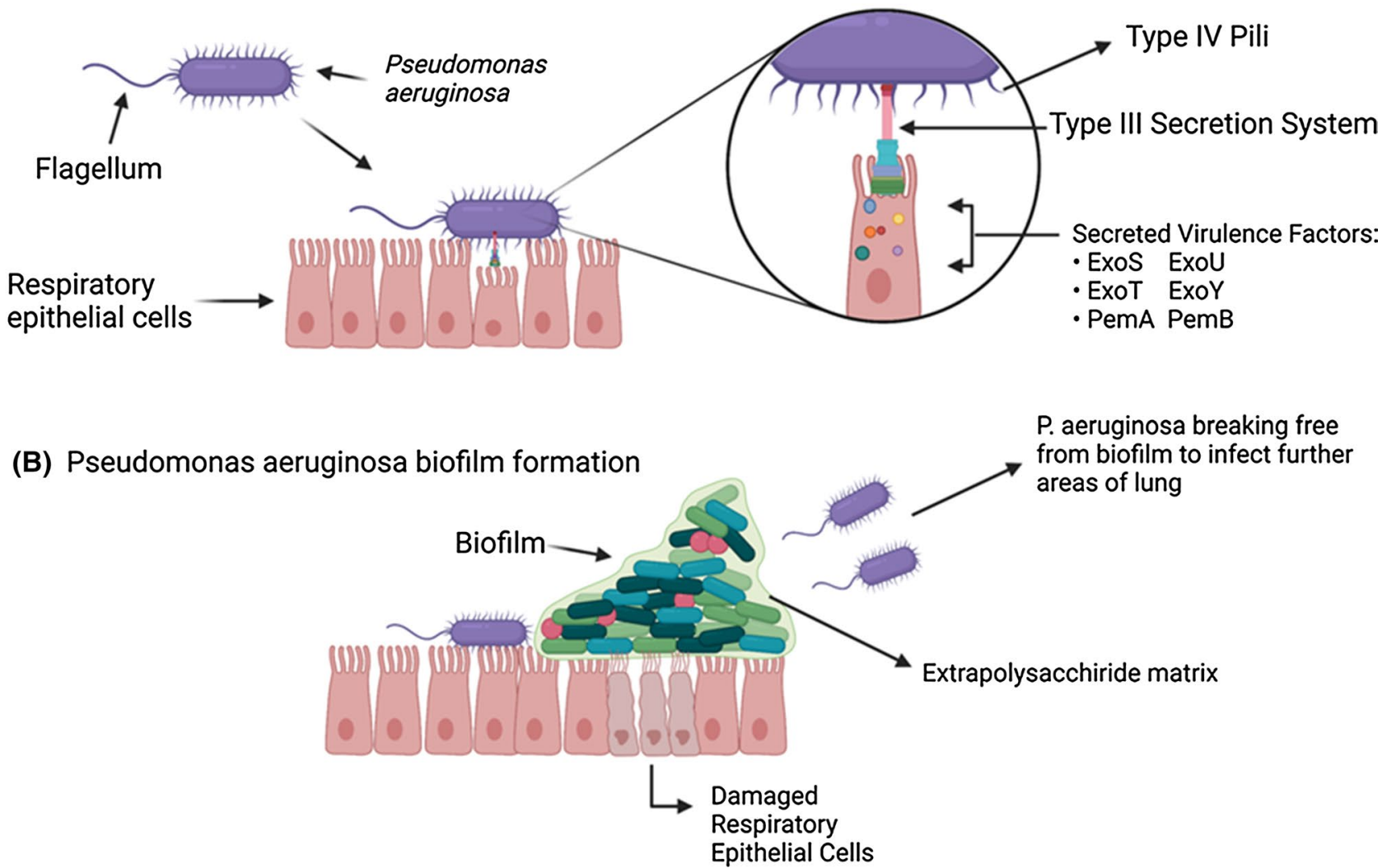

Fig. 1 Mechanisms of virulence and biofilm formation in Pseudomonas aeruginosa infections. Figure Created with BioRender.com

\section{Mechanisms of Infection, Virulence and Resistance}

\subsection{Motility and Attachment}

P. aeruginosa has flagella and pili that are necessary for motility and respiratory infection, as they enable attachment to respiratory epithelium via respiratory mucins and the glycolipid asialoGM1 $[54,55]$. Bacterial adhesion to the respiratory epithelium is an essential step for infection and is accomplished by interactions between bacterial adhesins and host receptors. For P. aeruginosa infection, the main adhesins are the single flagellum, which is necessary for motility, adhesion to cells and formation of a biofilm, and the type IV pili, which are appendages composed of pilin polymers, which allow the bacteria to move over surfaces in addition to serving relevant roles in biofilm formation and respiratory epithelial cell attachment [56]. Figure 1 outlines the relevant virulent mechanisms used by $P$. aeruginosa to attack respiratory epithelial cells.

\subsection{Biofilm Formation, Alginate Secretion, and Quorum Sensing}

Infection with $P$. aeruginosa can occur in an acute phase in patients with abnormal respiratory epithelium from patients with chronic lung disease or who have an acute illness that has led to respiratory failure and mechanical ventilation. A more chronic form of infection can occur in patients with underlying lung disease, such as CF. In the acute phase, $P$. aeruginosa is able to attach to respiratory epithelium using its type IV pili and flagellum, and toxins secreted by the bacterium damage the host cell lung. $P$. aeruginosa then begins to secrete an extracellular matrix, forming a biofilm, which is a structural matrix of bacterial cells encased within an extracellular matrix that adheres to the respiratory epithelium $[54,57]$. The matrix is largely made up of polysaccharides, proteins, extracellular DNA, and lipids. As a result of biofilm formation, bacteria are able to act synergistically while also providing protection from phagocytosis by neutrophils and antibiotics [54, 55, 57]. After the initial biofilm is formed, some bacteria may break free and progress to infect other areas of the lung (Fig. 1). Biofilm formation can also occur in the lungs of patients with chronic lung disease, 
such as in CF or chronic bronchiectasis, where $P$. aeruginosa organisms form a biofilm in thickened airway mucous and rarely need to travel to cell surfaces. In this model of chronic infection, $P$. aeruginosa bacteria lose their appendages and mutate into a mucoid phenotype with production of alginate, allowing for chronic infection [54].

Alginate is an exopolysaccharide composed of D-mannuronic acid and L-guluronic acid, and its production, induced by expression from $P$. aeruginosa, is essential for forming the mucoid phenotype of $P$. aeruginosa seen in CF. Typically, production of alginate is limited by an anti-sigma factor, MucA, which binds AlgT, preventing it from inducing expression of alginate at the algD promoter region. In the $\mathrm{CF}$ airway, $P$. aeruginosa acquires mutations in the mucA gene, which leads to uninhibited production of alginate due to unsuppressed activity of algT and conversion to a mucoid colony of $P$. aeruginosa [54, 58]. Alginate overproduction in $P$. aeruginosa is beneficial for the microbe for a variety of reasons including formation of biofilm, preventing bacterial clearance by host immune cells, and antimicrobial therapy, as well as immunomodulatory effects that may impair host immune response to infection [58].

Quorum sensing is a form of bacterial communication that enables $P$. aeruginosa to regulate gene expression allowing the microbes to coordinate activity against the host in the setting of infection. P. aeruginosa has several well-defined quorum sensing pathways, such as Ls, Rhl, and Pqs that all generate autoinducers, which diffuse into bacterial and host cells leading to transcriptional regulation that benefits continued bacterial survival and decreased immune response to infection $[55,59]$.

\subsection{Type III Secretions System}

Amongst the most powerful mechanisms for increasing the virulence and morbidity from a $P$. aeruginosa infection is the type III secretion system which enables the bacterium to inject effector proteins into host cells, such as the respiratory epithelium. The effector cells are able to alter host cell functions, such as disrupting innate immune response and altering host actin cytoskeleton [60]. Four effector proteins, ExoS, ExoT, ExoU, and ExoY have been typically described as the effector proteins used by the $P$. aeruginosa type III secretion system [61]; however, two additional effectors, PemA and PemB, have recently been described [62]. The most clinically relevant effectors may be ExoS and ExoU, which are thought to be mutually exclusive when found in $P$. aeruginosa strains [63]. ExoS impairs cell-to-cell adhesion through disruption of the actin cytoskeleton of host respiratory epithelial cells [63], while also inducing apoptosis of host cells [64]. ExoU is thought to be responsible for the greatest virulence due to its cytotoxic activity that induces host cell death and is more often found in patients in ICUs or burn units $[63,65,66]$.

Previous studies have demonstrated that the presence of a type III secretion system in $P$. aeruginosa is associated with poor outcomes including a larger burden of bacteria, persistence of infection and increased mortality [61, 63, 67-70]. Chronic infection with $P$. aeruginosa, such as in patients with CF, may select against type III secretion system, as many CF patients have antibodies directed against effector proteins and the conversion to a mucoid phenotype leading to downregulation of type III secretion proteins $[61,71,72]$. Given the relevance of the type III secretion system in acute infection, it is the subject of significant research to find targets for treatment to prevent or treat $P$. aeruginosa infection. $\mathrm{PcrV}$ is a part of the translocation apparatus and is necessary for injection of effector proteins into host cells, and immunization against PcrV has been shown to be protective against infection in animal studies [61]. P. aeruginosa isolates with PcrV have been associated with higher mortality, and clinical studies are ongoing to assess response to PcrV-specific antibodies [61, 68].

\subsection{Diagnosis of $P$. aeruginosa Infection}

The diagnosis of $P$. aeruginosa infection is dependent on timely and appropriate cultures obtained from an appropriate site. For critically ill patients with suspected $P$. aeruginosa infection, blood cultures should be obtained prior to starting antibiotics, ideally within one hour of identification of a critically ill patient [73]. Urine cultures should be obtained for patients with suspected UTI or CAUTI. For patients with suspected pneumonia, sputum cultures should be obtained when sputum is able to be produced. For patients with CF, sputum culture should be directed to look for CF-associated organisms, such as $P$. aeruginosa. The presence of $P$. aeruginosa on a sputum culture in a child can be an early clue to a diagnosis of CF [74].

Typical methods to identify $P$. aeruginosa in the laboratory include recognizing morphology and microbial colonies that have grown on media [75]. Selective media, such as that with cetrimide, is available and can be useful for identifying $P$. aeruginosa in polymicrobial samples [76]. Once identification of $P$. aeruginosa is made on culture, antimicrobial susceptibility testing is an essential next step in order to identify resistance and guide appropriate antibiotic therapy. Most labs use commercially available automated antimicrobial susceptibility kits that allow for identification of antimicrobial resistance as well as measurement of minimum inhibitory concentrations [75]. Identification of carbapenem-resistant strains of $P$. aeruginosa is essential, especially when identifying potential outbreaks.

Diagnosis of $P$. aeruginosa in nosocomial pneumonia presents more diagnostic challenge in terms of how to sample 
for the infection. In ventilated patients with suspected VAP or HAP that has subsequently led to mechanical ventilation, guidelines from the American Thoracic Society (ATS) and Infectious Disease Society of America (IDSA) recommend endotracheal aspiration for semiquantitative cultures, although quality evidence comparing endotracheal aspiration to more invasive sampling via a bronchoscopy with bronchoalveolar lavage (BAL) is lacking [6]. Guidelines from the European Respiratory Society (ERS) recommend obtaining more distal samples, such as via BAL in order to reduce unnecessary antibiotic exposure [77]. Proponents of invasive sampling via bronchoscopy with quantitative culture argue that more invasive sampling can lead to more precise therapy that targets the known pathogen and earlier narrowing of antibiotics. A study of 413 patients with VAP found that an invasive strategy was associated with more antibiotic-free days [78], whereas a subsequent study of 740 patients found no difference in antibiotic use between an invasive and non-invasive strategy [79]. Neither study showed a difference in 28-day mortality [78, 79].

Rapid diagnostic testing is also available for diagnosis of $P$. aeruginosa nosocomial pneumonia. Tests for lower respiratory tract infections that utilize multiplex real-time polymerase-chain reaction to test for a variety of pathogens known to cause pneumonia have demonstrated good sensitivity and specificity for $P$. aeruginosa, including BAL samples $[80,81]$. There are multiple advantages to utilizing a rapid diagnostic test for $P$. aeruginosa infection, including a diagnosis within $2 \mathrm{~h}$, as opposed to standard culture which can take 48-72 h, identification of concomitant viral or bacterial infection, and early identification of some antimicrobial resistance genes that are able to be identified in PCRtests that can lead to initial appropriate antibiotic therapy, as well as timely de-escalation of antibiotics [81]. An important caveat with rapid diagnostic testing is that, while it is able to assist in determining that the organism is present, it does not assist in determining if this is due to infection or colonization. Therefore, it is essential that it be ordered when there is concern for infection and results taken into clinical context.

\section{Treatment of $P$. aeruginosa Infections}

\subsection{Infection Control and Prevention}

One of the most important aspects of management of P. aeruginosa infections is preventing new infections whenever possible, especially in the setting of MDR organisms. There are a range of practices and guidelines available for preventing the spread of MDR pathogens like $P$. aeruginosa and other Gram-negative bacilli (GNB), including standard care such as hand hygiene and contact precautions for patients with MDR organisms [82-85]. Other relevant measures that may reduce the incidence of MDR P. aeruginosa include antimicrobial stewardship programs, environmental cleaning, and decolonization procedures [82]. Prevention of VAP, which is frequently due to $P$. aeruginosa, can be achieved with avoidance of intubation, when possible, daily assessments for extubation, minimizing sedation, elevating the head of the bed, and minimizing secretions. Furthermore, prevention of healthcare-associated infections such as CLABSI and CAUTI, as well as surgical site infections, can be achieved with strict sterile technique, frequent reassessments for the need of a central line or urinary catheter and removal of nonessential lines and catheter [86]. A large systematic review and meta-analysis previously found that the most effective measures for preventing the development of an MDR GNB infection in the ICU was a combination of standard care (hand hygiene and contact precautions), antimicrobial stewardship, environmental cleaning, and source control [82].

Given the prevalence of MDR P. aeruginosa organisms, overtreatment is a concern. Antibiotic de-escalation is an essential strategy when treating $P$. aeruginosa infections, and antibiotics should be narrowed once culture sensitivities have returned. Moreover, initial antimicrobial therapy directed against $P$. aeruginosa should only be done when there is a risk for $P$. aeruginosa infection. Additionally, certain antimicrobials that are available for MDR $P$. aeruginosa are often restricted by hospitals for use in known MDR $P$. aeruginosa and consultation with infectious disease department may be recommended in order to prescribe newer antimicrobials with MDR activity.

\subsection{Prompt and Appropriate Antibiotic Administration}

The initial management of a patient with a suspected $P$. aeruginosa infection, especially in those critically ill in the ICU, involves obtaining cultures and the administration of appropriate antibiotics. For patients who are ill with suspected sepsis, guidelines recommend administration of antibiotics within $1 \mathrm{~h} \mathrm{[87].} \mathrm{Additionally,} \mathrm{source} \mathrm{control} \mathrm{is} \mathrm{an} \mathrm{essential}$ part of treatment of any infection, and any abscesses should be drained while any indwelling devices such as urinary catheters or vascular catheters should be removed if thought to be the source of $P$. aeruginosa infection.

Appropriate initial antibiotic choice is essential in the management of $P$. aeruginosa infection, as inappropriate initial choice of antimicrobial regimen has previously been shown to be associated with increased mortality $[9,11,88$, 89]. A previous retrospective study found that initial inappropriate antimicrobial therapy in septic shock resulted in a 5 -fold reduction in survival [90]. A large meta-analysis of patients with severe bacterial infections showed that patients who received appropriate initial antibiotic therapy had lower 
rates of treatment failure, shorter hospital length of stay and cost, as well as reduced mortality rates compared to patients who received inappropriate initial antibiotic therapy [91]. Additional evidence for the benefits of appropriate initial therapy is seen in the MERINO trial, a prospective randomized trial that compared piperacillin-tazobactam to meropenem in patients with Gram-negative bacteremia that was ceftriaxone-resistant, which is typically treated with a carbapenem. In this noninferiority study, noninferiority could not be established for piperacillin-tazobactam compared to meropenem, with $12.3 \%$ of subjects randomized to piperacillin-tazobactam dying within 30 days, compared to $(3.7 \%)$ randomized to meropenem (risk difference, $8.6 \%$ [1-sided $97.5 \% \mathrm{CI},-\infty$ to $14.5 \%$ ]; $p=0.90$ for noninferiority) [92]. While the MERINO study was looking specifically into Klebsiella and E. coli organisms that were ceftriaxoneresistant, the trial demonstrates the importance of early appropriate antimicrobial therapy in severe bacterial infections, which also applies to $P$. aeruginosa infections.

Initial therapy with broad-spectrum antibiotics is often indicated, especially in critically ill patients while awaiting culture results [73]. Patients with known colonization or risk factors for MDR pathogens, should receive an initial antibiotic regimen that would be active against the MDR pathogen based on previous culture results or broad-spectrum antibiotics while awaiting culture results. Previous retrospective analyses have shown that initial antibiotics aimed at $P$. aeruginosa improve outcomes in healthcare-associated pneumonia and in nosocomial sepsis secondary to GNB $[9,93]$.

\subsection{Combination versus Monotherapy for Treatment of $P$. aeruginosa}

The management of $P$. aeruginosa infection with combination or monotherapy remains an area of debate. Given that inappropriate initial empiric therapy is a risk factor for mortality in $P$. aeruginosa infection, empiric therapy with a combination of antipseudomonal therapy is often given to severely ill patients $[6,73,75]$. Additionally, synergistic activity of a combination of antibiotics active against $P$. aeruginosa has been suggested to be beneficial in $P$. aeruginosa infection, although definitive evidence showing a clear benefit to synergistic activity is lacking [94-96].

Data regarding the benefits of combination therapy have been contradictory. In a retrospective single-center study of 100 patients with Pseudomonal bacteremia, inadequate initial antibiotics therapy was associated with increased mortality, and adequate combination therapy was associated with decreased mortality [97]. Additional observational and retrospective studies have suggested a potential benefit to combination therapy in patients with septic shock or VAP secondary to $P$. aeruginosa $[98,99]$. Conversely, a post hoc analysis of 593 patients with $P$. aeruginosa found no association with 30-day mortality in combination versus single drug therapy [100]. A meta-analysis comparing beta-lactam plus aminoglycoside or fluoroquinolone compared to beta-lactam monotherapy in $P$. aeruginosa infections also found no mortality benefit to using two active antipseudomonal agents over a single antibiotic as treatment [95]. Current guidelines for management of sepsis and septic shock recommend combination or multidrug therapy in critically ill patients with shock at risk of an organism that is associated with high rates of antimicrobial resistance, such as $P$. aeruginosa, in order to increase the likelihood that at least one active drug is being given [73]. A Cochrane review from 2016 found no difference in outcomes for CF patients treated with combination therapy compared to monotherapy in $P$. aeruginosa infections, although the studies included were at high risk of bias and combination therapy is still frequently used in CF exacerbations [96].

Management of VAP caused by $P$. aeruginosa also remains an area of contention, as guidelines state that there have been no randomized controlled trials which compare antibiotic regimens in patients with VAP caused by $P$. aeruginosa [6]. It is important to note that trials of HABP/VAP are difficult to complete for a variety of reasons including ensuring that patients enrolled have a nosocomial pneumonia, long time to completion of the study, and complexity surrounding novel antimicrobials being evaluated for both safety and efficacy in ill patients with a variety of pathogens [101]. Previous studies in HAP/VAP may have failed to show benefit due to inadequate dosing of antimicrobials in VAP in the setting of normal creatinine clearance and augmented renal clearance [101, 102].

\subsection{Treatment Recommendations for $P$. aeruginosa Nosocomial Pneumonia}

The most recent recommendations for treatment of $P$. aeruginosa in hospitalized patients make a distinction in the management of VAP versus hospital-acquired pneumonia (HAP). In the case of suspected VAP, joint recommendations from the IDSA and ATS state that empiric coverage including MRSA and anti-pseudomonal coverage is recommended in addition to coverage against other Gram-negative bacilli. Two antipseudomonal antibiotics, typically a betalactam and an aminoglycoside, are recommended in the management of VAP when the patient is at risk of multidrug resistance, patients in ICUs where $>10 \%$ of Gramnegative isolates are resistant to an agent being considered for monotherapy of a patient in an ICU where antimicrobial susceptibility rates are not known [6]. In the absence of these risk factors, a single antipseudomonal can be utilized in the treatment of VAP in addition to MRSA coverage. 
Table 1 Initial treatment of Pseudomonas aeruginosa pneumonia ${ }^{a}$

\begin{tabular}{|c|c|}
\hline \multicolumn{2}{|l|}{ One of the following } \\
\hline Ceftazidime & IV 2 g Q8-12 h \\
\hline Cefepime & IV $1-2$ g Q8 h \\
\hline Piperacillin-tazobactam & IV $4.5 \mathrm{~g}$ Q6 $\mathrm{h}$ \\
\hline Meropenem & IV $2 \mathrm{~g} \mathrm{Q8} \mathrm{h}$ \\
\hline Imipenem & IV $500 \mathrm{mg}$ Q6 h or $1 \mathrm{~g} \mathrm{Q} 8 \mathrm{~h}$ \\
\hline Aztreonam ${ }^{\mathrm{b}}$ & IV 2 g every $8 \mathrm{~h}$ \\
\hline \multicolumn{2}{|c|}{ Plus one of the following if treating with combination therapy } \\
\hline Tobramycin & IV $7 \mathrm{mg} / \mathrm{kg}$ od $^{\mathrm{c}}$ \\
\hline Gentamicin & IV $7 \mathrm{mg} / \mathrm{kg}$ od $^{\mathrm{c}}$ \\
\hline Levofloxacin & IV or PO $750 \mathrm{mg}$ od \\
\hline Ciprofloxacin & IV or PO $400 \mathrm{mg}$ od \\
\hline Amikacin & IV $20 \mathrm{mg} / \mathrm{kg}$ od $^{\mathrm{d}}$ \\
\hline \multicolumn{2}{|c|}{ Antimicrobial options for MDR-P. aeruginosa pneumonia } \\
\hline Ceftolozane-tazobactam & IV $3 \mathrm{~g} \mathrm{Q8} \mathrm{h}$ \\
\hline Ceftazidime-avibactam & IV $2.5 \mathrm{~g} \mathrm{Q} 8 \mathrm{~h}$ \\
\hline Imipenem-cilastatin-relebactam & IV $1.25 \mathrm{~g}$ Q6 h \\
\hline Cefiderocol & IV $2 \mathrm{~g} \mathrm{Q6}-8 \mathrm{~h}$ \\
\hline
\end{tabular}

$I V$ intravenous, $M D R$ multi-drug resistant, $o d$ once daily, $P O$ oral, $Q$ every

a Dosages recommended based on normal renal and hepatic function

${ }^{\mathrm{b}}$ Typically reserved for patients with beta-lactam allergy

${ }^{c}$ Dose should be adjusted for serum trough concentration $<1 \mu \mathrm{g} / \mathrm{mL}$

${ }^{\mathrm{d}}$ Dose should be adjusted for serum trough concentration $<5 \mu \mathrm{g} / \mathrm{mL}$

Table 2 Risk factors for Pseudomonas aeruginosa infection

Prior $P$. aeruginosa colonization

Structural lung disease (CF, bronchiectasis)

Hematological malignancy/neutropenia

Transplantation

Skin burns

Antimicrobial therapy within 90 days

Presence of indwelling catheter (vascular catheter, urinary catheter)

Prolonged hospitalization

Mechanical ventilation

$C F$ cystic fibrosis

In the case of HAP, similar antibiotic recommendations exist (see Table 1); however, combination therapy is only recommended in the setting of those at high risk for mortality (such as ventilated or those in septic shock) or those at high risk for drug resistance [6]. See Table 1 for specific antibiotic and dosing recommendations. Risk factors for $P$. aeruginosa infection, which may guide when to start broad-spectrum antibiotics or cover for MDR isolates, are outlined in Table 2.
Guidelines from European Societies differ from the ATS and IDSA. The European Respiratory Society (ERS) guidelines for the management of HAP/VAP recommend monotherapy with a narrow-spectrum antibiotic, such as ceftriaxone, ertapenem or levofloxacin for patients with suspected HAP/VAP deemed to be at low risk of MDR pathogens and low mortality risk. However, they recommend dual antipseudomonal coverage for patients with HAP/VAP with a high MDR risk and septic shock [77].

As mentioned, no specific RCTs have been conducted that specifically compared antimicrobial regimens for $P$. aeruginosa HAP/VAP. A meta-analysis that included 41 randomized trials with over 7000 patients found no specific antibiotic regimen was superior in reducing mortality and treatment failure in patients with VAP [103]. However, current guidelines do recommend against aminoglycosides as monotherapy in $P$. aeruginosa pneumonia due to poor lung penetration by aminoglycosides as well as lack of studies specifically investigating aminoglycosides in $P$. aeruginosa pneumonia. Furthermore, doripenem is not recommended in treatment of pneumonia as it currently holds a black-box warning [6] due to a clinical trial that found increased mortality and lower clinical cure rate than standard therapy in patients with VAP [102]. It is important to note that specific doses for nosocomial pneumonia secondary to $P$. aeruginosa may be higher than used in other circumstances. Poor penetration of antibiotics into alveolar space may contribute to the need for higher doses of antibiotics in order to penetrate epithelial lining fluid in the lung [101]. From a practical standpoint, antibiotics frequently used for the treatment of nosocomial pneumonia secondary to $P$. aeruginosa without evidence of MDR organisms typically include either cefepime, meropenem, piperacillin-tazobactam or imipenem. Specific reasons to include one antimicrobial over another may be dependent on patient factors such as allergies, renal function, as well as institutional factors such as which antibiotics are locally available as well as local antibiograms and resistance patterns.

\subsection{Other Treatment Options}

Ceftolozane-tazobactam is a novel combination antimicrobial and antipseudomonal activity. It has been approved for complicated UTIs and intraabdominal infections [6] with in vitro activity against $P$. aeruginosa isolates including drug-resistant and multi-drug resistant isolates [104]. A multi-center, non-inferiority RCT of ceftolozane-tazobactam in ventilated patients with nosocomial pneumonia demonstrated that ceftolozane-tazobactam was non-inferior to meropenem in 28-day mortality and clinical cure with an acceptable safety profile [105]. In this study, subjects received $3 \mathrm{~g}$ of IV ceftolozane-tazobactam, whereas the usual dose in UTI and intraabdominal infection is 1.5 
grams. Ceftolozane-tazobactam is now often used as monotherapy in $P$. aeruginosa infections known to be resistant to other beta-lactams, but is also increasingly being used in critically ill patients who have a high likelihood of having an MDR isolate of $P$. aeruginosa, as well as in high-risk patients who are known to be at risk for MDR $P$. aeruginosa, such as CF patients or lung transplant recipients [106]. Additionally, ceftolozane-tazobactam is increasingly recommended as empiric therapy in combination with an aminoglycoside in the management of nosocomial pneumonia in the ICU when there are risk factors for $P$. aeruginosa, known $P$. aeruginosa colonization, or high local incidence of $P$. aeruginosa. When $P$. aeruginosa is found to have caused pneumonia or bacteremia, ceftolozane-tazobactam monotherapy is recommended by some as targeted antimicrobial therapy due to risks of failure from MDR pathogens [107].

Ceftazidime-avibactam is another novel combination antimicrobial with antipseudomonal activity, combining the anti-pseudomonal cephalosporin ceftazidime with the non-beta-lactam beta-lactamase inhibitor avibactam, extending the antipseudomonal activity of ceftazidime. In a multicenter, non-inferiority RCT, ceftazidime-avibactam was found to be non-inferior to meropenem in the treatment of nosocomial pneumonia for the outcome of clinical cure [108]. In this study, the dose administered was $2 \mathrm{~g}$ of ceftazidime with $500 \mathrm{mg}$ of avibactam, administered intravenously. The addition of ceftolozane-tazobactam and ceftazidimeavibactam has increased the number of available drugs for the management of pneumonia due to $P$. aeruginosa, especially in drug-resistant isolates.

Another new antimicrobial available for use in MDR $P$. aeruginosa is imipenem-cilastatin-relebactam, which is a combination of carbapenem with relebactam, which is a beta-lactamase inhibitor that inhibits class A carbapenemases and class $C$ cephalosporinases [109-111]. A recent randomized multicenter trial compared imipenem-cilastatinrelebactam to piperacillin-tazobactam I adults with HAP or VAP and it was found to be noninferior [109]. Meropenem-vaborbactam is an additional combination carbapenem-beta-lactamase inhibitor, with activity against MDR $P$. aeruginosa [110, 111]. Data for the use of meropenemvaborbactam in pneumonia are lacking at this time, although it was found to be noninferior to piperacillin-tazobactam in a randomized study of complicated UTIs and acute pyelonephritis [112].

A newer antibiotic, cefiderocol, which is a novel siderophore cephalosporin, was recently studied in a multicenter randomized noninferiority study of 148 subjects, which compared cefiderocol to extended-infusion high-dose meropenem for subjects with hospital-acquired pneumonia or VAP due to GNB, and found that cefiderocol was noninferior to meropenem for the primary outcome of 14-day mortality, adding another potential choice for treatment of MDR pseudomonas in nosocomial pneumonia [113].

Infrequently used antimicrobials for MDR $P$. aeruginosa include plazomicin and colistin. Plazomicin is a synthetic derivative of sisomycin, and has activity against $P$. aeruginosa to a degree similar to that of other aminoglycosides, although data for its use in pneumonia due to $P$. aeruginosa are limited [111] Colistin, or polymyxin B, is an older antimicrobial with antipseudomonal activity in addition to good coverage against other MDR Gram-negative pathogens. While colistin is not used as frequently as other antimicrobials, it has been shown to be as efficacious as others in the management of VAP [114], although it does have a high incidence of nephrotoxicity, estimated at 18\%-43\% [115]. Colistin is also available as an inhaled antibiotic, and current guidelines recommend its use as an adjunctive therapy (in addition to IV therapy) in P. aeruginosa VAP when the isolate is only susceptible to aminoglycosides or polymyxins [6]. With the development of newer antimicrobials with strong activity against MDR $P$. aeruginosa, the use of colistin is not typically recommended as initial therapy in MDR $P$. aeruginosa.

As previously mentioned, use of newer antimicrobials with activity against MDR isolates of MDR $P$. aeruginosa are often restricted by hospitals and can only be prescribed in certain circumstances, typically the presence of MDR $P$. aeruginosa. The decision of what newer antimicrobial to use in the presence of an MDR organism depends on sensitivities from $P$. aeruginosa culture results as well as which antimicrobials are available to prescribe at a specific institution. Ceftolozane-tazobactam and ceftazidime-avibactam, as previously mentioned, are more commonly being used for treatment of MDR $P$. aeruginosa pneumonia, with options such as colistin rarely being used.

\subsection{Treatment of $P$. aeruginosa in Cystic Fibrosis Patients}

As previously mentioned, $P$. aeruginosa is the most important pathogen in patients with $\mathrm{CF}$ and up to $70 \%$ of $\mathrm{CF}$ patients will eventually develop chronic $P$. aeruginosa infection [116]. Chronic infection with $P$. aeruginosa in CF also frequently leads to conversion of $P$. aeruginosa to a mucoid phenotype [36, 37], and has been associated with worsening lung function and increased morbidity and mortality [116, 117]. Routine cultures are recommended in $\mathrm{CF}$ patients in order to identify early infection with $P$. aeruginosa [28].

New infection with $P$. aeruginosa that is identified in a CF patient should be treated in order to eradicate $P$. aeruginosa from the airways of patients with $\mathrm{CF}$, regardless of the presence of symptoms $[28,117,118]$. In an observational study of children with $\mathrm{CF}$ who developed a new P. aeruginosa infection, $69 \%$ were able to successfully achieve eradication 
of $P$. aeruginosa with antibiotic therapy, and those subjects who did achieve eradication had significantly lower risk of developing chronic $P$. aeruginosa infection and mucoid conversion over a 5-year follow-up period [118]. Eradication of $P$. aeruginosa has also been described to be successful in adults [119]. The Cystic Fibrosis Foundation guidelines strongly recommend eradication of initial $P$. aeruginosa infection [28].

The most typical regimen for treatment of initial P. aeruginosa in CF is inhaled tobramycin $300 \mathrm{mg}$ twice per day for 28 days, which has been shown to achieve eradication of P. aeruginosa in more than $70 \%$ of patients with associated improvements in lung function and reduced CF exacerbations [28, 32, 117-120]. Inhaled aztreonam has also been studied in new-onset $P$. aeruginosa infections, with eradication rates similar to inhaled tobramycin [121]. Furthermore, treatment with oral antibiotics such as ciprofloxacin in addition to inhaled antipseudomonas have not been more effective than inhaled tobramycin monotherapy [33, 122].

In addition to proper timing of antimicrobial therapy in $P$. aeruginosa infections, appropriate dosing of antibiotics is essential. Typical regimens used for the treatment of $P$. aeruginosa in the setting of normal renal function would include cefepime $2 \mathrm{~g}$ intravenously every $8 \mathrm{~h}$, meropenem 2 $\mathrm{g}$ intravenously every 8 hours, and piperacillin-tazobactam $4.5 \mathrm{~g}$ intravenously every 6 hours. It is important to note that antimicrobials used for $P$. aeruginosa are often used at higher doses and at increased frequency when compared to doses and frequencies used for same drug for other infectious organisms. Moreover, it is essential to monitor patient's renal function prior to and during use of antibiotics for $P$. aeruginosa infection, as dose adjustments are often necessary, and consultation with a pharmacist may be necessary.

Chronic infection with $P$. aeruginosa in CF patients is typically also managed with aerosolized antibiotics due to the progressive decline in lung function and increased mortality associated with chronic infection [117]. The most common regimen used in chronic $P$. aeruginosa infection in $\mathrm{CF}$ is a rotating 28 days of inhaled tobramycin followed by 28 days of no treatment. Inhaled tobramycin has been extensively studied in this setting and is associated with improvements in lung function and decreased CF exacerbations [120, 123-126]. Inhaled aztreonam, prescribed on a similar 28-days-on, 28-days-off regimen, is another treatment option for CF patients with chronic $P$. aeruginosa, and it has been shown to be efficacious in improving lung function, quality of life and time to further antibiotic therapy $[127,128]$, although it is not as extensively studied as tobramycin. Many patients who received inhaled aztreonam were previously treated with inhaled tobramycin [120]. Inhaled colistin is another aerosolized antipseudomonal that is an option for chronic infection, with studies demonstrating conflicting results regarding the superiority of tobramycin compared to
Table 3 Typical antibiotic regimen for acute cystic fibrosis exacerbation

\begin{tabular}{ll}
\hline Antibiotic & Dose \\
\hline Cefepime & IV $2 \mathrm{~g} \mathrm{Q8h}$ \\
Ceftazidime & IV $2 \mathrm{~g} \mathrm{Q8h}$ \\
Meropenem & IV $1 \mathrm{~g} \mathrm{Q8h}$ \\
Piperacillin-tazobactam & IV $4.5 \mathrm{~g} \mathrm{Q6h}$ \\
Plus & \\
Tobramycin & IV $10 \mathrm{mg} / \mathrm{kg} \mathrm{od}^{\mathrm{a}}$ \\
\hline
\end{tabular}

$I V$ intravenous, od once daily, $Q 6 h$ every $6 \mathrm{~h}, Q 8 h$ every $8 \mathrm{~h}$

${ }^{\text {a }}$ Dose should be adjusted to a serum peak concentration of 20-30 $\mathrm{mg} / \mathrm{L}$ and a trough concentration of $<1 \mathrm{mg} / \mathrm{L}$

colistin, and current guidelines do not recommend inhaled colistin [124, 129, 130].

Acute exacerbations of $\mathrm{CF}$ are defined by clinical changes of a CF patent including changes in sputum, hemoptysis, worsening cough or dyspnea, fever, decrements in lung function, and radiographic changes, amongst other possible symptoms [131]. CF exacerbations are especially relevant in the management of CF patients, as they decrease lung function, worsen quality of life, and are associated with significant costs due to hospitalization [131]. The management of acute bronchiectasis exacerbations in non-CF patients is often similar and understanding the treatment of a CF exacerbation is important for physicians in multiple specialties. Most CF exacerbations are thought to be due to bacteria, with pseudomonas being the most important pathogen in CF. Therefore, management of a CF exacerbation involves intravenous antibiotics with antipseudomonal activity, and current guidelines recommend combination therapy with two intravenous antibiotics with activity against pseudomonas for moderate-to-severe exacerbations [94]. These recommendations are largely based on expert opinion and quality evidence comparing outcomes for single therapy versus combination therapy in CF exacerbations is lacking [96].

Specific antibiotic recommendations that are typically used at Barnes-Jewish Hospital are outlined in Table 3. Aminoglycosides, such as tobramycin, are commonly used for management of $\mathrm{CF}$ exacerbations and current recommendations are for once-daily dosing of aminoglycosides, which may reduce nephrotoxicity [94, 132]. Beta-lactam antibiotics such as piperacillin-tazobactam, cefepime, ceftazidime, or meropenem, are typical antibiotics used in addition to an aminoglycoside. It is important to note specific dose recommendations for $P$. aeruginosa in Table 3 , as CF patients are known to have altered pharmacokinetics, due to alterations in volume of distribution of certain antibiotics in nutritionally deficient $\mathrm{CF}$ patients, metabolism and clearance, while also facing challenges in lung penetration of intravenous antibiotics due to altered lung architecture [133]. Optimal 
duration of antimicrobial therapy in a $\mathrm{CF}$ exacerbation remains an area of debate, and while current guidelines do not recommend a specific time frame, treatment often ranges from as few as 10 days to up to 3 weeks [94].

\section{Conclusion}

P. aeruginosa is an important Gram-negative pathogen, particularly in patients with chronic lung disease and in patients at risk for nosocomial infections. It is a particularly important pathogen in hospital-acquired and ventilator-associated nosocomial pneumonias. P. aeruginosa possesses a number of virulence factors that enable it to attack respiratory epithelial cells and is adept at developing mechanisms for antimicrobial resistance. Treatment of $P$. aeruginosa will often involve combination therapy with two antibiotics which are known to have activity against pseudomonas; this appropriate early antibiotic therapy in $P$. aeruginosa infections is associated with improved outcomes. Exacerbations of CF will also typically be treated with antimicrobials with activity against $P$. aeruginosa, due to the high rates of chronic infection with $P$. aeruginosa in patients with CF. Newer antibiotics have been developed for treatment of MDR P. aeruginosa, such as ceftolozane-tazobactam, which is increasingly being used as therapy for patients with suspected MDR $P$. aeruginosa.

\section{Declarations}

Funding None.

Conflicts of interest None to declare.

Ethics approval Not applicable.

Informed consent Not applicable.

Data availability Not applicable.

\section{References}

1. Magill SS, et al. Multistate point-prevalence survey of health care-associated infections. N Engl J Med. 2014;370(13):1198208. https://doi.org/10.1056/NEJMoa1306801.

2. Weiner LM, et al. Antimicrobial-resistant pathogens associated with healthcare-associated infections: summary of data reported to the National Healthcare Safety Network at the Centers for Disease Control and Prevention, 2011-2014. Infect Control Hosp Epidemiol. 2016;37(11):1288-301. https://doi.org/10.1017/ice. 2016.174.

3. Williams BJ, Dehnbostel J, Blackwell TS. Pseudomonas aeruginosa: host defence in lung diseases. Respirology.
2010;15(7):1037-56. https://doi.org/10.1111/j.1440-1843.2010. 01819.x.

4. Parker CM, et al. Ventilator-associated pneumonia caused by multidrug-resistant organisms or Pseudomonas aeruginosa: prevalence, incidence, risk factors, and outcomes. J Crit Care. 2008;23(1):18-26. https://doi.org/10.1016/j.jcrc.2008.02.001.

5. Vincent JL, et al. Prevalence and outcomes of infection among patients in intensive care units in 2017. JAMA. 2020;323(15):1478-87. https://doi.org/10.1001/jama.2020.2717.

6. Kalil AC, et al. Management of Adults With Hospital-acquired and Ventilator-associated Pneumonia: 2016 Clinical Practice Guidelines by the Infectious Diseases Society of America and the American Thoracic Society. Clin Infect Dis. 2016;63(5):e61111. https://doi.org/10.1093/cid/ciw353.

7. Fujitani S, et al. Pneumonia due to Pseudomonas aeruginosa: part I: epidemiology, clinical diagnosis, and source. Chest. 2011;139(4):909-19. https://doi.org/10.1378/chest.10-0166.

8. Planquette B, et al. Pseudomonas aeruginosa ventilator-associated pneumonia. Predictive factors of treatment failure. Am J Respir Crit Care Med. 2013;188(1):69-76. https://doi.org/10. 1164/rccm.201210-18970C.

9. Micek ST, Reichley RM, Kollef MH. Health care-associated pneumonia (HCAP): empiric antibiotics targeting methicillinresistant Staphylococcus aureus (MRSA) and Pseudomonas aeruginosa predict optimal outcome. Medicine (Baltimore). 2011;90(6):390-5. https://doi.org/10.1097/MD.0b013e3182 39cf0a.

10. Trouillet JL, et al. Pseudomonas aeruginosa ventilator-associated pneumonia: comparison of episodes due to piperacillin-resistant versus piperacillin-susceptible organisms. Clin Infect Dis. 2002;34(8):1047-54. https://doi.org/10.1086/339488.

11. Micek ST, et al. Pseudomonas aeruginosa nosocomial pneumonia: impact of pneumonia classification. Infect Control Hosp Epidemiol. 2015;36(10):1190-7. https://doi.org/10.1017/ice.2015. 167.

12. Kollef MH, et al. Global prospective epidemiologic and surveillance study of ventilator-associated pneumonia due to Pseudomonas aeruginosa. Crit Care Med. 2014;42(10):2178-87. https://doi.org/10.1097/CCM.0000000000000510.

13. Bergin SP, et al. PROPHETIC: prospective identification of pneumonia in hospitalized patients in the ICU. Chest. 2020;158(6):2370-80. https://doi.org/10.1016/j.chest.2020.06. 034.

14. Rosenthal VD, et al. International Nosocomial Infection Control Consortium report, data summary of 50 countries for 2010-2015: device-associated module. Am J Infect Control. 2016;44(12):1495-504. https://doi.org/10.1016/j.ajic.2016.08. 007.

15. Mittal R, et al. Urinary tract infections caused by Pseudomonas aeruginosa: a minireview. J Infect Public Health. 2009;2(3):10111. https://doi.org/10.1016/j.jiph.2009.08.003.

16. Kitagawa K, et al. Bacteremia complicating urinary tract infection by Pseudomonas aeruginosa: mortality risk factors. Int $\mathrm{J}$ Urol. 2019;26(3):358-62. https://doi.org/10.1111/iju.13872.

17. Nicolle LE. Catheter associated urinary tract infections. Antimicrob Resist Infect Control. 2014;3:23. https://doi.org/10.1186/ 2047-2994-3-23.

18. Venier AG, et al. Nosocomial urinary tract infection in the intensive care unit: when should Pseudomonas aeruginosa be suspected? Experience of the French national surveillance of nosocomial infections in the intensive care unit, Rea-Raisin. Clin Microbiol Infect. 2012;18(1):E13-5. https://doi.org/10.1111/j. 1469-0691.2011.03686.x.

19. Elgohari S, et al. Impact of national policies on the microbial aetiology of surgical site infections in acute NHS hospitals in England: analysis of trends between 2000 and 2013 
using multi-centre prospective cohort data. Epidemiol Infect. 2017;145(5):957-69. https://doi.org/10.1017/S09502688160030 58 .

20. Massart N, et al. Mortality due to hospital-acquired infection after cardiac surgery. J Thorac Cardiovasc Surg. 2020. https:// doi.org/10.1016/j.jtcvs.2020.08.094.

21. Norbury W, et al. Infection in Burns. Surg Infect (Larchmt). 2016;17(2):250-5. https://doi.org/10.1089/sur.2013.134.

22. Williams FN, et al. The leading causes of death after burn injury in a single pediatric burn center. Crit Care. 2009;13(6):R183. https://doi.org/10.1186/cc8170.

23. Mayhall CG. The epidemiology of burn wound infections: then and now. Clin Infect Dis. 2003;37(4):543-50. https://doi.org/10. 1086/376993.

24. Azzopardi EA, et al. Gram negative wound infection in hospitalised adult burn patients-systematic review and metanalysis. PLoS ONE. 2014;9(4):e95042. https://doi.org/10.1371/journal. pone.0095042.

25. Kang CI, et al. Bloodstream infections caused by antibioticresistant Gram-negative bacilli: risk factors for mortality and impact of inappropriate initial antimicrobial therapy on outcome. Antimicrob Agents Chemother. 2005;49(2):760-6. https://doi. org/10.1128/AAC.49.2.760-766.2005.

26. Micek ST, et al. Resistance to empiric antimicrobial treatment predicts outcome in severe sepsis associated with Gram-negative bacteremia. J Hosp Med. 2011;6(7):405-10. https://doi.org/10. 1002/jhm.899.

27. Thaden JT, et al. Results from a 13-year prospective cohort study show increased mortality associated with bloodstream infections caused by Pseudomonas aeruginosa compared to other bacteria. Antimicrob Agents Chemother. 2017. https://doi.org/10.1128/ AAC.02671-16.

28. Mogayzel PJ Jr, et al. Cystic Fibrosis Foundation pulmonary guideline. Pharmacologic approaches to prevention and eradication of initial Pseudomonas aeruginosa infection. Ann Am Thorac Soc. 2014;11(10):1640-50. https://doi.org/10.1513/ AnnalsATS.201404-1660C.

29. Sanders DB, et al. Failure to recover to baseline pulmonary function after cystic fibrosis pulmonary exacerbation. Am J Respir Crit Care Med. 2010;182(5):627-32. https://doi.org/10.1164/ rccm.200909-14210C.

30. Jacques I, et al. Pulmonary evolution of cystic fibrosis patients colonized by Pseudomonas aeruginosa and/or Burkholderia cepacia. Eur J Pediatr. 1998;157(5):427-31. https://doi.org/10. 1007/s004310050844.

31. Saiman L, Siegel J, P. Cystic Fibrosis Foundation Consensus Conference on Infection Control. Infection control recommendations for patients with cystic fibrosis: Microbiology, important pathogens, and infection control practices to prevent patient-topatient transmission. Am J Infect Control, 2003. 31(3 Suppl): S1-62.

32. Ratjen F, et al. Treatment of early Pseudomonas aeruginosa infection in patients with cystic fibrosis: the ELITE trial. Thorax. 2010;65(4):286-91. https://doi.org/10.1136/thx.2009.121657.

33. Treggiari MM, et al. Comparative efficacy and safety of 4 randomized regimens to treat early Pseudomonas aeruginosa infection in children with cystic fibrosis. Arch Pediatr Adolesc Med. 2011;165(9):847-56. https://doi.org/10.1001/archpediatrics. 2011.136

34. Saiman L, et al. Infection prevention and control guideline for cystic fibrosis: 2013 update. Infect Control Hosp Epidemiol. 2014;35(Suppl 1):S1-67. https://doi.org/10.1086/676882.

35. Smyth AR, et al. European cystic fibrosis society standards of care: best practice guidelines. J Cyst Fibros. 2014;13(Suppl 1):S23-42. https://doi.org/10.1016/j.jcf.2014.03.010.
36. Langan KM, Kotsimbos T, Peleg AY. Managing Pseudomonas aeruginosa respiratory infections in cystic fibrosis. Curr Opin Infect Dis. 2015;28(6):547-56. https://doi.org/10.1097/QCO. 0000000000000217.

37. Malhotra S, Hayes D Jr, Wozniak DJ. Mucoid Pseudomonas aeruginosa and regional inflammation in the cystic fibrosis lung. J Cyst Fibros. 2019;18(6):796-803. https://doi.org/10.1016/j.jcf. 2019.04.009.

38. Li Z, et al. Longitudinal development of mucoid Pseudomonas aeruginosa infection and lung disease progression in children with cystic fibrosis. JAMA. 2005;293(5):581-8. https://doi.org/ 10.1001/jama.293.5.581.

39. Salsgiver EL, et al. Changing epidemiology of the respiratory bacteriology of patients with cystic fibrosis. Chest. 2016;149(2):390-400. https://doi.org/10.1378/chest.15-0676.

40. Hatziagorou E, et al. Changing epidemiology of the respiratory bacteriology of patients with cystic fibrosis-data from the European cystic fibrosis society patient registry. J Cyst Fibros. 2020;19(3):376-83. https://doi.org/10.1016/j.jcf.2019.08.006.

41. Shoemark A, Ozerovitch L, Wilson R. Aetiology in adult patients with bronchiectasis. Respir Med. 2007;101(6):1163-70. https:// doi.org/10.1016/j.rmed.2006.11.008.

42. Loebinger MR, et al. Mortality in bronchiectasis: a long-term study assessing the factors influencing survival. Eur Respir J. 2009;34(4):843-9. https://doi.org/10.1183/09031936.00003709.

43. Woo TE, et al. Epidemiology and natural history of Pseudomonas aeruginosa airway infections in non-cystic fibrosis bronchiectasis. ERJ Open Res. 2018. https://doi.org/10.1183/23120541. 00162-2017.

44. Bilton D. Update on non-cystic fibrosis bronchiectasis. Curr Opin Pulm Med. 2008;14(6):595-9. https://doi.org/10.1097/MCP. 0b013e328312ed8c.

45. Tofas $\mathrm{P}$, et al. Pseudomonas aeruginosa bacteraemia in patients with hematologic malignancies: risk factors, treatment and outcome. Diagn Microbiol Infect Dis. 2017;88(4):335-41. https:// doi.org/10.1016/j.diagmicrobio.2017.05.003.

46. Cattaneo C, et al. P. aeruginosa bloodstream infections among hematological patients: an old or new question? Ann Hematol. 2012;91(8):1299-304. https://doi.org/10.1007/ s00277-012-1424-3.

47. Carvalho AS, et al. Bloodstream infections in neutropenic patients with haematological malignancies. Infect Dis Health. 2020;25(1):22-9. https://doi.org/10.1016/j.idh.2019.08.006.

48. Johnson LE, et al. Pseudomonas aeruginosa bacteremia over a 10-year period: multidrug resistance and outcomes in transplant recipients. Transpl Infect Dis. 2009;11(3):227-34. https://doi. org/10.1111/j.1399-3062.2009.00380.x.

49. CDC. Multidrug-resistant Pseudomonas aeruginosa. 2021. https://arpsp.cdc.gov/profile/antibiotic-resistance/mdr-empse udomonas-aeruginosaem.

50. Weiner-Lastinger LM, et al. Antimicrobial-resistant pathogens associated with adult healthcare-associated infections: summary of data reported to the National Healthcare Safety Network, 2015-2017. Infect Control Hosp Epidemiol. 2020;41(1):1-18. https://doi.org/10.1017/ice.2019.296.

51. Walters MS, et al. Carbapenem-resistant Pseudomonas aeruginosa at US emerging infections program sites, 2015. Emerg Infect Dis. 2019;25(7):1281-8. https://doi.org/10.3201/eid2507. 181200 .

52. Wong PH, et al. Antimicrobial co-resistance patterns of Gramnegative bacilli isolated from bloodstream infections: a longitudinal epidemiological study from 2002-2011. BMC Infect Dis. 2014;14:393. https://doi.org/10.1186/1471-2334-14-393.

53. Micek ST, et al. An international multicenter retrospective study of Pseudomonas aeruginosa nosocomial pneumonia: impact of 
multidrug resistance. Crit Care. 2015;19:219. https://doi.org/10. 1186/s13054-015-0926-5.

54. Lau GW, Hassett DJ, Britigan BE. Modulation of lung epithelial functions by Pseudomonas aeruginosa. Trends Microbiol. 2005;13(8):389-97. https://doi.org/10.1016/j.tim.2005.05.011.

55. Curran CS, Bolig T, Torabi-Parizi P. Mechanisms and Targeted Therapies for Pseudomonas aeruginosa Lung Infection. Am J Respir Crit Care Med. 2018;197(6):708-27. https://doi.org/10. 1164/rccm.201705-1043SO.

56. Bucior I, Pielage JF, Engel JN. Pseudomonas aeruginosa pili and flagella mediate distinct binding and signaling events at the apical and basolateral surface of airway epithelium. PLoS Pathog. 2012;8(4):e1002616. https://doi.org/10.1371/journal.ppat.10026 16.

57. Maurice NM, Bedi B, Sadikot RT. Pseudomonas aeruginosa biofilms: host response and clinical implications in lung infections. Am J Respir Cell Mol Biol. 2018;58(4):428-39. https://doi.org/ 10.1165/rcmb.2017-0321TR.

58. Hauser AR, et al. Clinical significance of microbial infection and adaptation in cystic fibrosis. Clin Microbiol Rev. 2011;24(1):2970. https://doi.org/10.1128/CMR.00036-10.

59. Papenfort K, Bassler BL. Quorum sensing signal-response systems in Gram-negative bacteria. Nat Rev Microbiol. 2016;14(9):576-88. https://doi.org/10.1038/nrmicro.2016.89.

60. Deng W, et al. Assembly, structure, function and regulation of type III secretion systems. Nat Rev Microbiol. 2017;15(6):32337. https://doi.org/10.1038/nrmicro.2017.20.

61. Hauser AR. The type III secretion system of Pseudomonas aeruginosa: infection by injection. Nat Rev Microbiol. 2009;7(9):65465. https://doi.org/10.1038/nrmicro2199.

62. Burstein D, et al. Novel type III effectors in Pseudomonas aeruginosa. MBio. 2015;6(2):e00161. https://doi.org/10.1128/mBio. 00161-15.

63. Horna G, Ruiz J. Type 3 secretion system of Pseudomonas aeruginosa. Microbiol Res. 2021;246: 126719. https://doi.org/10. 1016/j.micres.2021.126719.

64. Kaminski A, et al. Pseudomonas aeruginosa ExoS induces intrinsic apoptosis in target host cells in a manner that is dependent on its GAP domain activity. Sci Rep. 2018;8(1):14047. https://doi. org/10.1038/s41598-018-32491-2.

65. Bradbury RS, et al. Virulence gene distribution in clinical, nosocomial and environmental isolates of Pseudomonas aeruginosa. J Med Microbiol. 2010;59(Pt 8):881-90. https://doi.org/10.1099/ jmm.0.018283-0.

66. Horna $\mathrm{G}$, et al. High frequency of the exoU+/exoS+ genotype associated with multidrug-resistant "high-risk clones" of Pseudomonas aeruginosa clinical isolates from Peruvian hospitals. Sci Rep. 2019;9(1):10874. https://doi.org/10.1038/ s41598-019-47303-4.

67. Hauser AR, et al. Type III protein secretion is associated with poor clinical outcomes in patients with ventilator-associated pneumonia caused by Pseudomonas aeruginosa. Crit Care Med. 2002;30(3):521-8. https://doi.org/10.1097/00003246-20020 3000-00005.

68. Roy-Burman A, et al. Type III protein secretion is associated with death in lower respiratory and systemic Pseudomonas aeruginosa infections. J Infect Dis. 2001;183(12):1767-74. https:// doi.org/10.1086/320737.

69. Zhuo H, et al. Increased mortality of ventilated patients with endotracheal Pseudomonas aeruginosa without clinical signs of infection. Crit Care Med. 2008;36(9):2495-503. https://doi.org/ 10.1097/CCM.0b013e318183f3f8.

70. El Solh AA, et al. Persistent infection with Pseudomonas aeruginosa in ventilator-associated pneumonia. Am J Respir Crit Care Med. 2008;178(5):513-9. https://doi.org/10.1164/rccm. 200802-239OC.
71. Moss J, et al. Sera from adult patients with cystic fibrosis contain antibodies to Pseudomonas aeruginosa type III apparatus. Infect Immun. 2001;69(2):1185-8. https://doi.org/10.1128/IAI. 69.2.1185-1188.2001.

72. Wu W, et al. MucA-mediated coordination of type III secretion and alginate synthesis in Pseudomonas aeruginosa. J Bacteriol. 2004;186(22):7575-85. https://doi.org/10.1128/JB.186.22.75757585.2004.

73. Rhodes A, et al. Surviving sepsis campaign: international guidelines for management of sepsis and septic shock: 2016. Crit Care Med. 2017;45(3):486-552. https://doi.org/10.1097/CCM.00000 00000002255.

74. Farrell PM, et al. Guidelines for diagnosis of cystic fibrosis in newborns through older adults: cystic fibrosis foundation consensus report. J Pediatr. 2008;153(2):S4-14. https://doi.org/10. 1016/j.jpeds.2008.05.005.

75. Bassetti M, et al. How to manage Pseudomonas aeruginosa infections. Drugs Context. 2018;7:212527. https://doi.org/10. 7573/dic.212527.

76. Kodaka $\mathrm{H}$, et al. Evaluation of a new agar medium containing cetrimide, kanamycin and nalidixic acid for isolation and enhancement of pigment production of Pseudomonas aeruginosa in clinical samples. J Basic Microbiol. 2003;43(5):407-13. https://doi.org/10.1002/jobm.200310264.

77. Torres A, et al. International ERS/ESICM/ESCMID/ALAT guidelines for the management of hospital-acquired pneumonia and ventilator-associated pneumonia: Guidelines for the management of hospital-acquired pneumonia (HAP)/ventilator-associated pneumonia (VAP) of the European Respiratory Society (ERS), European Society of Intensive Care Medicine (ESICM), European Society of Clinical Microbiology and Infectious Diseases (ESCMID) and Asociacion Latinoamericana del Torax (ALAT). Eur Respir J. 2017. https://doi.org/10.1183/13993003. 00582-2017.

78. Fagon JY, et al. Invasive and noninvasive strategies for management of suspected ventilator-associated pneumonia. A randomized trial. Ann Intern Med. 2000;132(8):621-30. https://doi. org/10.7326/0003-4819-132-8-200004180-00004.

79. Canadian Critical Care Trials G. A randomized trial of diagnostic techniques for ventilator-associated pneumonia. N Engl J Med. 2006;355(25):2619-30. https://doi.org/10.1056/NEJMoa052904.

80. Murphy CN, et al. Multicenter evaluation of the BioFire FilmArray pneumonia/pneumonia plus panel for detection and quantification of agents of lower respiratory tract infection. J Clin Microbiol. 2020. https://doi.org/10.1128/JCM.00128-20.

81. Kollef MH, Burnham CD. Ventilator-associated pneumonia: the role of emerging diagnostic technologies. Semin Respir Crit Care Med. 2017;38(3):253-63. https://doi.org/10.1055/s-0037-15992 24.

82. Teerawattanapong $\mathrm{N}$, et al. Prevention and control of multidrugresistant gram-negative bacteria in adult intensive care units: a systematic review and network meta-analysis. Clin Infect Dis. 2017;64(suppl_2):S51-60. https://doi.org/10.1093/cid/cix112.

83. Tacconelli E, et al. ESCMID guidelines for the management of the infection control measures to reduce transmission of multidrug-resistant Gram-negative bacteria in hospitalized patients. Clin Microbiol Infect. 2014;20(Suppl 1):1-55. https://doi.org/ 10.1111/1469-0691.12427.

84. Siegel JD, et al. 2007 Guideline for Isolation Precautions: Preventing Transmission of Infectious Agents in Health Care Settings. Am J Infect Control. 2007;35(10 Suppl 2):S65-164. https:// doi.org/10.1016/j.ajic.2007.10.007.

85. Siegel JD, et al. Management of multidrug-resistant organisms in health care settings, 2006. Am J Infect Control. 2007;35(10 Suppl 2):S165-93. https://doi.org/10.1016/j.ajic.2007.10.006. 
86. Yokoe DS, et al. A compendium of strategies to prevent healthcare-associated infections in acute care hospitals: 2014 updates. Infect Control Hosp Epidemiol. 2014;35(Suppl 2):S21-31. https://doi.org/10.1017/s0899823x00193833.

87. Levy MM, Evans LE, Rhodes A. The surviving sepsis campaign bundle: 2018 update. Crit Care Med. 2018;46(6):997-1000. https://doi.org/10.1097/CCM.0000000000003119.

88. Micek ST, et al. Health care-associated pneumonia and community-acquired pneumonia: a single-center experience. Antimicrob Agents Chemother. 2007;51(10):3568-73. https://doi.org/ 10.1128/AAC.00851-07.

89. Micek ST, et al. Pseudomonas aeruginosa bloodstream infection: importance of appropriate initial antimicrobial treatment. Antimicrob Agents Chemother. 2005;49(4):1306-11. https://doi. org/10.1128/AAC.49.4.1306-1311.2005.

90. Kumar A, et al. Initiation of inappropriate antimicrobial therapy results in a fivefold reduction of survival in human septic shock. Chest. 2009;136(5):1237-48. https://doi.org/10.1378/ chest.09-0087.

91. Bassetti M, et al. Systematic review of the impact of appropriate versus inappropriate initial antibiotic therapy on outcomes of patients with severe bacterial infections. Int J Antimicrob Agents. 2020;56(6):106184. https://doi.org/10.1016/j.ijantimicag.2020.106184.

92. Harris PNA, et al. Effect of Piperacillin-Tazobactam vs Meropenem on 30-day mortality for patients with E. coli or Klebsiella pneumoniae Bloodstream Infection and ceftriaxone resistance: a randomized clinical trial. JAMA. 2018;320(10):984-94. https://doi.org/10.1001/jama.2018. 12163.

93. Micek ST, et al. Empiric combination antibiotic therapy is associated with improved outcome against sepsis due to Gram-negative bacteria: a retrospective analysis. Antimicrob Agents Chemother. 2010;54(5):1742-8. https://doi.org/10.1128/AAC.01365-09.

94. Flume PA, et al. Cystic fibrosis pulmonary guidelines: treatment of pulmonary exacerbations. Am J Respir Crit Care Med. 2009;180(9):802-8. https://doi.org/10.1164/rccm. 200812-1845PP.

95. Vardakas KZ, et al. beta-Lactam plus aminoglycoside or fluoroquinolone combination versus beta-lactam monotherapy for Pseudomonas aeruginosa infections: a meta-analysis. Int J Antimicrob Agents. 2013;41(4):301-10. https://doi.org/10.1016/j. ijantimicag.2012.12.006.

96. Elphick HE, Scott A. Single versus combination intravenous antipseudomonal antibiotic therapy for people with cystic fibrosis. Cochrane Database Syst Rev. 2016;12:CD002007. https://doi. org/10.1002/14651858.CD002007.pub4.

97. Park SY, et al. Impact of adequate empirical combination therapy on mortality from bacteremic Pseudomonas aeruginosa pneumonia. BMC Infect Dis. 2012;12:308. https://doi.org/10.1186/ 1471-2334-12-308.

98. Kumar A, et al. Early combination antibiotic therapy yields improved survival compared with monotherapy in septic shock: a propensity-matched analysis. Crit Care Med. 2010;38(9):177385. https://doi.org/10.1097/CCM.0b013e3181eb3ced.

99. Garnacho-Montero J, et al. Optimal management therapy for Pseudomonas aeruginosa ventilator-associated pneumonia: an observational, multicenter study comparing monotherapy with combination antibiotic therapy. Crit Care Med. 2007;35(8):188895. https://doi.org/10.1097/01.CCM.0000275389.31974.22.

100. Pena $\mathrm{C}$, et al. Effect of adequate single-drug vs combination antimicrobial therapy on mortality in Pseudomonas aeruginosa bloodstream infections: a post Hoc analysis of a prospective cohort. Clin Infect Dis. 2013;57(2):208-16. https://doi.org/10. 1093/cid/cit223.
101. Kollef MH, Micek ST. Limitations of registration trials for nosocomial pneumonia. Clin Infect Dis. 2020. https://doi.org/ 10.1093/cid/ciaa926.

102. Kollef $\mathrm{MH}$, et al. A randomized trial of 7-day doripenem versus 10-day imipenem-cilastatin for ventilator-associated pneumonia. Crit Care. 2012;16(6):R218. https://doi.org/10.1186/cc11862.

103. Aarts MA, et al. Empiric antibiotic therapy for suspected ventilator-associated pneumonia: a systematic review and meta-analysis of randomized trials. Crit Care Med. 2008;36(1):108-17. https:// doi.org/10.1097/01.CCM.0000297956.27474.9D.

104. Carvalhaes CG, et al. Antimicrobial activity of ceftolozanetazobactam tested against Gram-negative contemporary (20152017) isolates from hospitalized patients with pneumonia in US medical centers. Diagn Microbiol Infect Dis. 2019;94(1):93-102. https://doi.org/10.1016/j.diagmicrobio.2018.11.021.

105. Kollef MH, et al. Ceftolozane-tazobactam versus meropenem for treatment of nosocomial pneumonia (ASPECT-NP): a randomised, controlled, double-blind, phase 3, non-inferiority trial. Lancet Infect Dis. 2019;19(12):1299-311. https://doi.org/10. 1016/S1473-3099(19)30403-7.

106. Lizza BD, et al. New perspectives on antimicrobial agents: Ceftolozane-Tazobactam. Antimicrob Agents Chemother. 2021;65(7):e0231820. https://doi.org/10.1128/AAC.02318-20.

107. Zaragoza R, et al. Update of the treatment of nosocomial pneumonia in the ICU. Crit Care. 2020;24(1):383. https://doi.org/10. 1186/s13054-020-03091-2.

108. Torres A, et al. Ceftazidime-avibactam versus meropenem in nosocomial pneumonia, including ventilator-associated pneumonia (REPROVE): a randomised, double-blind, phase 3 noninferiority trial. Lancet Infect Dis. 2018;18(3):285-95. https:// doi.org/10.1016/S1473-3099(17)30747-8.

109. Titov I, et al. A randomized, double-blind, multicenter trial comparing efficacy and safety of Imipenem/Cilastatin/Relebactam Versus Piperacillin/Tazobactam in adults with hospital-acquired or ventilator-associated bacterial pneumonia (RESTORE-IMI 2 Study). Clin Infect Dis. 2020. https://doi.org/10.1093/cid/ciaa8 03.

110. Zhanel GG, et al. Imipenem-Relebactam and MeropenemVaborbactam: two novel carbapenem-beta-lactamase inhibitor combinations. Drugs. 2018;78(1):65-98. https://doi.org/10.1007/ s40265-017-0851-9.

111. Doi Y. Treatment options for carbapenem-resistant gram-negative bacterial infections. Clin Infect Dis. 2019;69(Suppl 7):S565-75. https://doi.org/10.1093/cid/ciz830.

112. Kaye KS, et al. Effect of Meropenem-Vaborbactam vs Piperacillin-Tazobactam on clinical cure or improvement and microbial eradication in complicated urinary tract infection: The TANGO I Randomized Clinical Trial. JAMA. 2018;319(8):788-99. https:// doi.org/10.1001/jama.2018.0438.

113. Wunderink RG, et al. Cefiderocol versus high-dose, extendedinfusion meropenem for the treatment of Gram-negative nosocomial pneumonia (APEKS-NP): a randomised, double-blind, phase 3, non-inferiority trial. Lancet Infect Dis. 2021;21(2):21325. https://doi.org/10.1016/S1473-3099(20)30731-3.

114. Florescu DF, et al. What is the efficacy and safety of colistin for the treatment of ventilator-associated pneumonia? A systematic review and meta-regression. Clin Infect Dis. 2012;54(5):670-80. https://doi.org/10.1093/cid/cir934.

115. Barbier F, et al. Hospital-acquired pneumonia and ventilatorassociated pneumonia: recent advances in epidemiology and management. Curr Opin Pulm Med. 2013;19(3):216-28. https:// doi.org/10.1097/MCP.0b013e32835f27be.

116. Crull MR, et al. Changing rates of chronic Pseudomonas aeruginosa infections in cystic fibrosis: a population-based cohort study. Clin Infect Dis. 2018;67(7):1089-95. https://doi.org/10. 1093/cid/ciy215. 
117. Flume PA, et al. Cystic fibrosis pulmonary guidelines: chronic medications for maintenance of lung health. Am J Respir Crit Care Med. 2007;176(10):957-69. https://doi.org/10.1164/rccm. 200705-664OC.

118. Mayer-Hamblett N, et al. Impact of sustained eradication of new Pseudomonas aeruginosa infection on long-term outcomes in cystic fibrosis. Clin Infect Dis. 2015;61(5):707-15. https://doi. org/10.1093/cid/civ377.

119. Kenny SL, et al. Eradication of Pseudomonas aeruginosa in adults with cystic fibrosis. BMJ Open Respir Res. 2014;1(1):e000021. https://doi.org/10.1136/bmjre sp-2014-000021.

120. Smith S, Rowbotham NJ, Regan KH. Inhaled anti-pseudomonal antibiotics for long-term therapy in cystic fibrosis. Cochrane Database Syst Rev. 2018;3:CD001021. https://doi.org/10.1002/ 14651858.CD001021.pub3.

121. Tiddens HA, et al. Open label study of inhaled aztreonam for Pseudomonas eradication in children with cystic fibrosis: The ALPINE study. J Cyst Fibros. 2015;14(1):111-9. https://doi.org/ 10.1016/j.jcf.2014.06.003.

122. Langton Hewer SC, Smyth AR. Antibiotic strategies for eradicating Pseudomonas aeruginosa in people with cystic fibrosis. Cochrane Database Syst Rev. 2017;4:CD004197. https://doi.org/ 10.1002/14651858.CD004197.pub5.

123. Gibson RL, et al. Significant microbiological effect of inhaled tobramycin in young children with cystic fibrosis. Am J Respir Crit Care Med. 2003;167(6):841-9. https://doi.org/10.1164/ rccm.200208-855OC.

124. Mogayzel PJ Jr, et al. Cystic fibrosis pulmonary guidelines. Chronic medications for maintenance of lung health. Am J Respir Crit Care Med. 2013;187(7):680-9. https://doi.org/10. 1164/rccm.201207-1160oe.

125. Cystic Fibrosis F, et al. Cystic fibrosis foundation evidencebased guidelines for management of infants with cystic fibrosis.
J Pediatr. 2009;155(6 Suppl):S73-93. https://doi.org/10.1016/j. jpeds.2009.09.001.

126. Lahiri $\mathrm{T}$, et al. Clinical practice guidelines from the cystic fibrosis foundation for preschoolers with cystic fibrosis. Pediatrics. 2016. https://doi.org/10.1542/peds.2015-1784.

127. McCoy KS, et al. Inhaled aztreonam lysine for chronic airway Pseudomonas aeruginosa in cystic fibrosis. Am J Respir Crit Care Med. 2008;178(9):921-8. https://doi.org/10.1164/rccm. 200712-1804OC.

128. Retsch-Bogart GZ, et al. Efficacy and safety of inhaled aztreonam lysine for airway pseudomonas in cystic fibrosis. Chest. 2009;135(5):1223-32. https://doi.org/10.1378/chest.08-1421.

129. Adeboyeku D, Scott S, Hodson ME. Open follow-up study of tobramycin nebuliser solution and colistin in patients with cystic fibrosis. J Cyst Fibros. 2006;5(4):261-3. https://doi.org/ 10.1016/j.jcf.2006.05.009.

130. Assael BM, et al. Inhaled aztreonam lysine vs. inhaled tobramycin in cystic fibrosis: a comparative efficacy trial. J Cyst Fibros. 2013;12(2):130-40. https://doi.org/10.1016/j.jcf.2012.07.006.

131. Bilton D, et al. Pulmonary exacerbation: towards a definition for use in clinical trials. Report from the EuroCareCF Working Group on outcome parameters in clinical trials. J Cyst Fibros. 2011. 10(Suppl 2):S79-S81. https://doi.org/10.1016/S15691993(11)60012-X.

132. Smyth A, et al. Once versus three-times daily regimens of tobramycin treatment for pulmonary exacerbations of cystic fibrosis-the TOPIC study: a randomised controlled trial. Lancet. 2005;365(9459):573-8. https://doi.org/10.1016/S0140-6736(05) 17906-9.

133. Akkerman-Nijland AM, et al. The pharmacokinetics of antibiotics in cystic fibrosis. Expert Opin Drug Metab Toxicol. 2021;17(1):53-68. https://doi.org/10.1080/17425255.2021. 1836157. 